TRANS · NúM.II $\cdot 2007$

DOSSIER $\cdot 155-169$
En este artículo se describen los principales tipos de discapacidad que pueden sufrir los usuarios y que afectan a su uso de las Tecnologias de la Información y Comunicación (TIC), y las dificultades que suponen a la hora de utilizar programas informáticos. Se analizarán las ayudas técnicas desarrolladas para superar estas dificultades, asi como las pautas, guias y técnicas dictadas por diversos organismos, nacionales e internacionales, para el diseño de tecnología accesible. Por último se hará un breve repaso de la principal normativa y legislación relativa a la accesibilidad en las TIC, y una relación entre los conceptos de accesibilidad y usabilidad.

PALABRAS CLAVE: Discapacidad, accesibilidad, usabilidad, TIC, ayudas técnicas, legislación, diseño para todos.

\title{
La accesibilidad y las tecnologías en la información y la comunicación
}

M. a Luz Guenaga, Ander Barbier \& Andoni Eguiluz Universidad de Deusto
This article describes the main kinds of difficulties users with disabilities may have with regard to Information and Communication Technologies (ICT), and the drawbacks they may experience when using computer programs. The article analyzes the assistive technology which has been developed to overcome these difficulties, and also describes the guidelines and techniques offered by different national and international organizations to design accessible technology. Finally, the article summarizes the main existing norms and laws related to accessibility in ICT, and the relationship between accessibility and usability.

KEYWORDS: Disability, accessibility, usability, ICT, assistive technology, law, design for all. 


\section{INTRODUCCIÓN: ACCESIBILIDAD Y TIC}

En el ámbito de las TIC, la accesibilidad se define como «el arte de garantizar que cualquier recurso, a través de cualquier medio, esté disponible para todas las personas, tengan o no algún tipo de discapacidad» (Berners-Lee y Fischetti 1999). Son muchas las discapacidades que pueden limitar el uso de las Tic, como por ejemplo la discapacidad motora de uno o varios miembros que dificulta el correcto uso de dispositivos como el ratón o el teclado, o la discapacidad visual que impide el acceso a toda la información visualizada en la pantalla de un ordenador. Como principal solución a estos problemas existen herramientas, tanto hardware como software, que sustituyen la funcionalidad que no tienen los usuarios con discapacidad. Como ejemplo de estas ayudas técnicas o tecnologías asistidas, se pueden mencionar los teclados y ratones adaptados, lectores de pantalla que leen en Voz alta los contenidos que se visualizan en la pantalla, sistemas de reconocimiento de voz, etc.

Para garantizar el acceso a cualquier recurso tecnológico a todas las personas, incluidas las que sufren algún tipo de discapacidad, no es suficiente con desarrollar tecnologías asistidas adecuadas para cada tipo de discapacidad. También es necesario que los propios recursos tecnológicos estén diseñados para que puedan ser accedidos a través de estas ayudas técnicas. Imaginemos a un usuario con una discapacidad que le impide utilizar el ratón, por lo que únicamente utiliza un teclado especial como dispositivo de entrada. Este usuario no podrá acceder a aplicaciones informáticas en las que haya operaciones que sólo se puedan llevar a cabo con el ratón. Existen pautas y recomendaciones para el diseño y desarrollo de aplicaciones y contenidos accesibles, que facilitan la labor de los desarrolladores de software y de los editores

de contenidos interesados en que sus productos sean accesibles.

En el mundo físico se han desarrollado muchos elementos para facilitar la vida a las personas con discapacidad, como rampas de acceso, baños adaptados, puertas y ascensores con medidas especiales, etc. Con el paso del tiempo, se ha demostrado que estos elementos también son beneficiosos para personas que no sufren ningún tipo de discapacidad, como los padres que llevan coches de niños, los transportistas que mueven bultos grandes, o las personas mayores. De la misma forma, en el mundo tecnológico, también se ha demostrado que los recursos accesibles son más fáciles y cómodos de utilizar por todos los usuarios, incluidos los que no sufren ninguna discapacidad.

La accesibilidad a las nuevas tecnologías preocupa a muchas empresas, organismos e instituciones. Proporcionar servicios y contenido accesible permite llegar a un mayor número de usuarios, aumentar los clientes potenciales, involucrar a las empresas en un proceso de concienciación social y beneficiar, principalmente, a usuarios con diversos tipos y grados de discapacidad, pero desgraciadamente, no todos los agentes implicados en la creación de servicios y contenidos lo ven así, y no todos pueden asumir los costes adicionales que ello conlleva. Por este motivo, es fundamental que la legislación garantice un nivel mínimo de accesibilidad en las Tics.

\section{TIPOS DE DISCAPACIDAD}

Son muchos y muy diversos los tipos de discapacidad que pueden afectar al acceso a las Tic. Puede variar la gravedad de las mismas, ser permanentes, temporales o crónicas, pueden ser de nacimiento o a lo largo de la vida, y pueden padecerse varias discapacidades a la vez. $\mathrm{La}$ Organización Mundial de la Salud publicó en 
200I una exhaustiva clasificación de las discapacidades (oms 200I), que en lo que se refiere a las TICs se suelen agrupar en discapacidades sensoriales, cognitivas, motoras y tecnológicas.

\section{I Discapacidad sensorial}

La discapacidad sensorial que en mayor medida afecta a las TIC es la visual y la auditiva, que suele presentarse a lo largo de la vida, y se agudiza en personas mayores.

La discapacidad visual es una de las que mayores limitaciones presenta a la hora de acceder a un mundo como el tecnológico, que es eminentemente gráfico. Cuando Internet, y las interfaces en general, eran únicamente en modo texto, la información era neutra, y se podía representar igualmente en modo audio, texto o Braille (Vanderheiden 1998). Las modernas interfaces de usuario, basadas en ventanas, iconos, imágenes, vídeos y animaciones, impiden que la información y servicios lleguen adecuadamente a las personas que presentan baja visión, ceguera total, daltonismo, vista cansada, cataratas u otros de los múltiples problemas de visión existentes.

La otra gran discapacidad sensorial es la auditiva. Puede variar desde los problemas de comprensión o audición hasta la sordera total. En contra de lo que muchas veces se cree, el mayor problema de las personas con discapacidad auditiva no es el hecho en sí de no oír correctamente, sino los problemas de comunicación y comprensión derivados de no haber desarrollado el lenguaje del mismo modo que las personas oyentes.

Otro tipo de discapacidades sensoriales temporales son, por ejemplo, las derivadas de trabajar en un ambiente ruidoso, tener la vista ocupada en una tarea como conducir o manejar una máquina, llevar auriculares para aislarse del ruido o no llevar lentes correctoras en el momento necesario. El uso de las TIC en cualquiera de estas circunstancias hace necesario el diseño y el uso de tecnologías accesibles.

\subsection{Discapacidad motora}

Son muchos y muy diversos los tipos de discapacidad motora o física que pueden limitar el acceso a las Tic, la más problemática es, sin embargo, la que afecta a las extremidades superiores, que dificultan o impiden el uso de los dispositivos periféricos como el ratón o el teclado.

La discapacidad motora puede deberse a malformaciones genéticas, enfermedades degenerativas, accidentes o a la progresiva falta de movilidad y precisión en los movimientos de las personas mayores.

\subsection{Problemas del habla}

No son muchas las interfaces que se manejan a través del habla, o por reconocimiento de voz, pero sí hay líneas de investigación y cada vez más productos comerciales que la utilizan. Las personas con dificultad en el habla encontrarán dificultades a la hora de manejar este tipo de interfaces, si no se les proporcionan otras alternativas.

\subsection{Discapacidad cognitiva}

El grupo de usuarios con este tipo de discapacidad es uno de los más numerosos y variado, ya que incluye a personas con problemas de dislexia, de comprensión gráfica o escrita, daños neuronales, retrasos emocionales y de desarrollo psíquico, déficit en la capacidad de concentración o memoria, enfermedades degenerativas como la enfermedad de Alzheimer, etc.

Las personas con discapacidad cognitiva tienen problemas a la hora de leer y comprender el contenido ofrecido a través de las TIC, y 
también con la forma de interactuar y utilizar programas informáticos que les pueden resultar muy complejos.

158

\section{AYUdAS TÉCNICAS}

Las ayudas técnicas permiten o facilitan el acceso a las TIC a las personas con discapacidad. Existen herramientas hardware y software (ATRC) que son de uso específico para un colectivo y otras que benefician a un grupo más amplio de usuarios.

Las primeras ayudas técnicas, o tecnología para la rehabilitación (Abascal I998), surgen de iniciativas personales y artesanales, de personas con amigos o familiares que sufren algún tipo de discapacidad, y que ven en la tecnología una solución a sus problemas.

A continuación se describen algunas de las ayudas técnicas más utilizadas.

\section{I Lector de pantalla}

Una de las ayudas más utilizadas es el lector de pantalla, un programa software que transforma el contenido textual de la pantalla y lo sintetiza en voz. Permite configurar aspectos como el idioma, para mejorar la pronunciación, y la velocidad de lectura, ya que los usuarios, a medida que van cogiendo práctica con el lector, son capaces de entender y leer a mayor velocidad.

Dos de los lectores más utilizados son JaWs (Freedom Scientific 2003) y Windows Eyes (GWM 2006). Son programas de uso general, capaces de leer cualquier aplicación del sistema compatible con esta tecnología. Otros lectores, como IBM Home Page Reader (Івм 2006), son programas específicos para la lectura de páginas web.

Los lectores de pantalla son especialmente útiles para las personas con discapacidad visual severa, a las que transmiten toda la información que no pueden ver. A las personas con discapacidad cognitiva les complementa la información que llega a través de otros canales, y les ayuda a comprender mejor el contenido si además de poder leerlo ellos mismos, se lo lee el sistema.

El problema de los lectores de pantalla es que si hay información no textual como imágenes, gráficos, iconos o vídeos, ésta no puede transmitirse mediante voz. Es necesario que haya una equivalente textual o sonora para que esta información llegue al usuario, de lo contrario resultará «invisible».

\subsection{Magnificador de pantalla}

Es un programa software que amplifica cierta zona de la pantalla, generalmente la que tiene el foco del cursor, como si fuera una lupa. Permite, a personas con baja visión, aumentar el tamaño del área visualizada. Algunos sistemas integran además lectores de pantalla, opciones para configurar el tipo y tamaño de letra, contraste o color, que complementan su funcionalidad.

El problema al utilizar el magnificador de pantalla es que se pierde información de contexto, ya que el área visualizada es muy pequeña. Se puede entender este problema reduciendo el tamaño de la ventana a una octava parte, aproximadamente, de su tamaño original: esta es la información que perciben los usuarios de los magnificadores. Para contrarrestar esta desventaja se suelen utilizar pantallas de mayor tamaño.

\subsection{Sistemas de reconocimiento de voz}

Estos programas permiten introducir comandos o información al sistema a través de la voz, como alternativa o complemento al ratón y al teclado. Algunos sistemas están diseñados para reconocer un amplio conjunto de voces diferentes, pero en estos casos el conjunto de comandos reconocido suele estar más limitado. Otros reconocedores, por el contrario, reconocen muchas órdenes, palabras y frases, de unos 


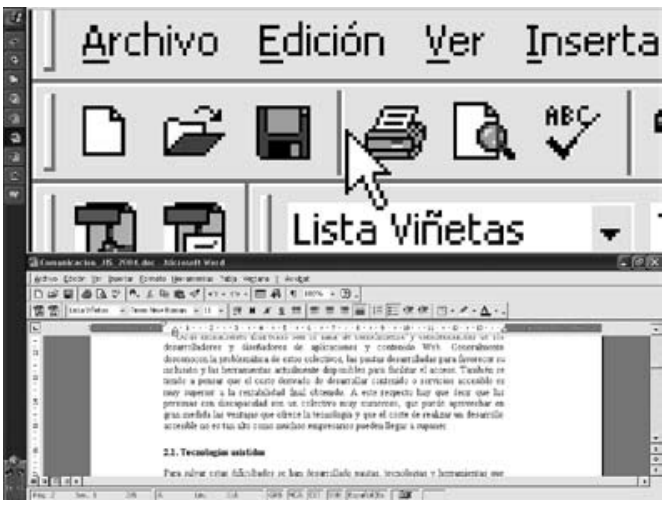

Fig. I.- Ejemplo de amplificación de pantalla

pocos usuarios que previamente han entrenado al sistema. Uno de los programas más utilizados es Via Voice de гвм (гым 2006).

Las personas con problemas de visión, y más aún las que sufren discapacidad motora severa, son los principales usuarios de los programas reconocedores de voz, ya que reduce la necesidad del uso del ratón o del teclado.

\subsection{Linea de Braille}

Las líneas de Braille son dispositivos hardware en los que dinámicamente se forman los caracteres Braille del contenido textual de la pantalla (IBRC 2006). Pueden representar entre I2 y 80 caracteres al mismo tiempo, y disponen además de un conjunto reducido de teclas que permiten navegar por pantalla, tabular hacia atrás o hacia delante, avanzar o retroceder en la lectura, y en algunos casos introducir caracteres mediante ocho teclas que representan los ocho puntos de los caracteres Braille.

Además del elevado precio de estos dispositivos, un inconveniente a la hora de utilizarlos es que no todas las personas ciegas saben leer Braille. La mayor parte de las cegueras no son de nacimiento, ni se producen en los primeros años de vida, sino que son derivadas de enfer-
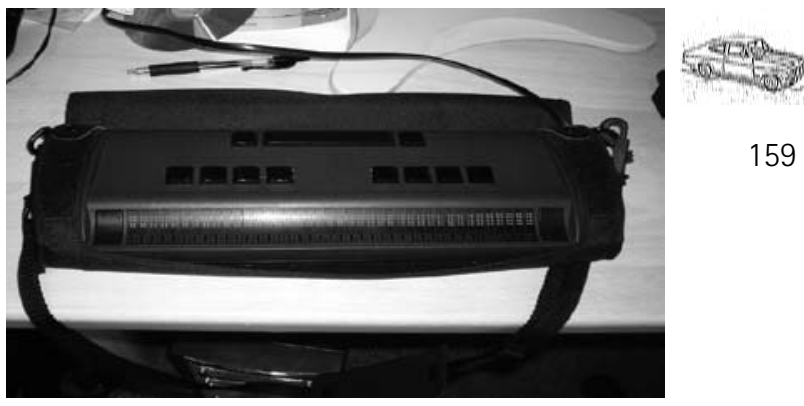

159

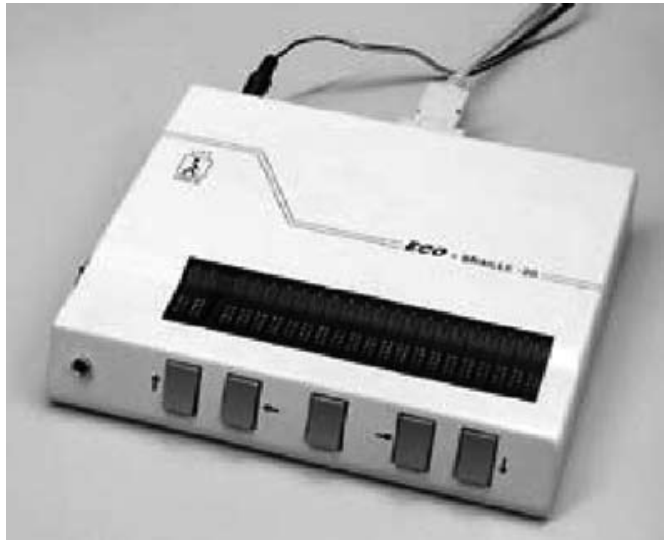

Fig. 2+3.- Lineas de Braille

medades progresivas o accidentes a lo largo de la vida. Esto hace que los usuarios no tengan desarrollada la sensibilidad necesaria en los dedos para leer caracteres Braille con fluidez. Pueden reconocer caracteres individuales, como el piso en un ascensor o el número de una puerta, pero no un texto extenso.

\subsection{Navegador sólo texto}

Las personas con discapacidad visual suelen utilizar navegadores sólo texto (ISC 2006), que extraen toda la información textual del contenido, facilitando la labor de los lectores de pantalla y de las líneas de Braille. Al igual que éstos 
últimos, si existe información no textual, resulta invisible para los usuarios que utilizan este tipo de navegadores.

160 Los diseñadores y desarrolladores de páginas web también utilizan los navegadores sólo texto para hacerse una idea de cómo percibirán esas páginas los usuarios que utilicen lectores de pantalla, líneas de Braille o navegadores sólo texto.

\subsection{Emulador de teclado}

Los usuarios con discapacidad motora, y todos aquellos que tengan dificultad en utilizar el teclado, pueden utilizar emuladores software de teclado, que muestran en pantalla un teclado que se puede manejar con dispositivos de entrada alternativos o con unas pocas teclas del teclado estándar.

\subsection{Pantalla táctil}

No sólo los programas y herramientas diseñadas para personas con discapacidad están utilizando cada vez más las pantallas táctiles, sino que el uso de este tipo de dispositivos se está abriendo paso en muchos de los servicios y sistemas de uso cotidiano, como los cajeros automáticos, dispensadores de títulos de transporte, venta de entradas y puntos de información turística.

Si la interfaz está bien diseñada, la selección de opciones suele resultar muy sencilla y requiere únicamente tocar la zona activa, lo que resulta muy útil para personas con dificultad o limitación en el movimiento y en general para todos los usuarios que requieren un dispositivo de entrada alternativo al que les causa problemas.

Para la introducción de texto se puede proporcionar un teclado adicional o uno simulado en pantalla, también activo mediante el tacto.

\subsection{Filtros de teclado}

$\mathrm{E} 1$ sistema $\mathrm{T}_{9}$ de los teléfonos móviles (Tepic 2006) es un buen ejemplo de filtro de teclado.
Esta tecnología se basa en un diccionario interno e inteligencia artificial, y permite escribir mensajes cortos (Short Message System - SMS) con tan solo presionar una tecla por letra en el teléfono.

Los filtros de teclado incluyen ayudas para la escritura, sistemas que predicen la palabra que se está escribiendo, y revisores ortográficos que reducen el número de pulsaciones de teclado necesarias para introducir texto, también reducen la posibilidad de pulsar teclas de forma involuntaria y de cometer errores.

\subsection{Dispositivos de entrada alternativos}

Existe un gran número de dispositivos de entra$\mathrm{da}$, principalmente teclados y ratones, adaptados para personas con discapacidad motora. Teclados ampliados o reducidos, ratones de gran tamaño o que se manejan con la boca o con los pies, teclados para una sola mano, soportes para el brazo que reducen el temblor y aumentan la precisión del movimiento y apuntadores de muy diversos tipos, son algunos de los dispositivos desarrollados para facilitar la introducción de datos y la interacción con el ordenador.

\section{Io Herramientas de validación y reparación}

Hay otra serie de herramientas, como los validadores y los reparadores, que ayudan al diseño y desarrollo de contenido y servicios accesibles, principalmente orientados a la tecnología Web. No constituyen ayudas técnicas en sí mismos, pero son de gran utilidad a la hora de diseñar productos tecnológicos siguiendo las pautas existentes.

Las «herramientas de validación» sirven para detectar problemas de accesibilidad en tiempo de desarrollo, y se basan generalmente en las pautas de la Iniciativa de Accesibilidad Web (Web Accessibility Initiative - WAI), que se comenta más adelante (WAI 2006). Algunos sistemas de validación se centran en aspectos 
concretos de la accesibilidad, mientras que otros, más generales, proporcionan información global sobre la accesibilidad de las páginas web.

Estas herramientas realizan un análisis estático de la página web y generan un informe que suele incluir, como mínimo, el problema detectado, su nivel de prioridad, una descripción detallada del mismo y el lugar en el que se ha detectado.

Se distinguen dos tipos de problemas, los errores, que es necesario corregir para obtener el nivel de conformidad deseado, y los avisos, que son aspectos difíciles de evaluar automáticamente, y que el autor debe revisar manualmente. Entre estos últimos se encuentra por ejemplo, la claridad y sencillez del contenido o la correcta estructuración del mismo. Algunas de las herramientas de validación comerciales más utilizadas son Bobby (Watc 2002) o AccVerify (HiSo 2006). Otras herramientas gratuitas son Hera (Sidar 2006) y el Test de Accesibilidad Web (cTIC 2006).
Los «reparadores», además de indicar el error producido, proporcionan pautas y ayudas para corregirlo. Los autores de páginas web no tienen por qué ser expertos en accesibilidad, por lo que se les debe proporcionar toda la ayuda y las herramientas necesarias para poder crear servicios y productos web accesibles. Entre los reparadores más utilizados destacan $A$-prompt (ATRC 2006) y AccRepair (HiSo I996).

También existen herramientas de validación de código HTML (Hypertext Markup Language) y Hojas de Estilo en Cascada (Cascading Style Sheets - css), que detectan las diferencias del código con respecto al estándar dictado por el Consorcio Mundial Web (World Wide Web Consortium - w3 $)\left(\mathrm{W}_{3} \mathrm{CV}\right.$ 2002).

\section{RELACIÓN ENTRE AYUDAS TÉCNICAS Y DISCAPACIDADES}

En la tabla que se presenta a continuación, se puede apreciar la relación entre las ayudas técnicas descritas y el tipo de discapacidad a los que beneficia principalmente:

\begin{tabular}{|l|c|c|c|c|}
\hline & \multicolumn{2}{|c|}{ SENSORIAL } & \multirow{2}{*}{ Motora } & \multirow{2}{*}{ Cognitiva } \\
\cline { 2 - 5 } & VISUAL & AUditiva & & $\mathrm{X}$ \\
\hline Lector de pantalla & $\mathrm{X}$ & & & $\mathrm{X}$ \\
\hline Magnificador de pantalla & $\mathrm{X}$ & & & $\mathrm{X}$ \\
\hline Reconocedor de voz & & & $\mathrm{X}$ & \\
\hline Línea de Braille & $\mathrm{X}$ & & & \\
\hline Navegador sólo texto & $\mathrm{X}$ & & & $\mathrm{X}$ \\
\hline Emulador de teclado & & & $\mathrm{X}$ & $\mathrm{X}$ \\
\hline Pantalla táctil & & & $\mathrm{X}$ & $\mathrm{X}$ \\
\hline Filtros de teclado & & & $\mathrm{X}$ & $\mathrm{X}$ \\
\hline $\begin{array}{l}\text { Dispositivos de entrada } \\
\text { alternativos }\end{array}$ & & & $\mathrm{X}$ & \\
\hline
\end{tabular}

Tabla I: Relación entre ayudas técnicas y tipo de discapacidad 


\section{NORMATIVA}

Las publicaciones recientes sobre accesibilidad describen múltiples principios, pautas y reco-

162 mendaciones para el diseño y desarrollo de productos accesibles. Los principios de diseño son generalmente objetivos de alto nivel, las pautas reflejan los requisitos de una organización o caso en particular, y las recomendaciones suelen ser instrucciones concretas acerca de la interacción con los elementos.

Son muchas las pautas que se han desarrollado para proporcionar el escenario en el que las personas con discapacidad puedan acceder a las TIC, y más concretamente a la información y servicios ofrecidos a través de Internet. Estas pautas no sólo benefician a las personas con discapacidad, también las personas que sufren discapacidad temporal y el conjunto global de usuarios perciben una mejora general de la usabilidad de los servicios y páginas web.

Las pautas de accesibilidad crean el marco necesario para hacer que las páginas o servicios a través de Internet sean accesibles; por su parte, las ayudas técnicas proporcionan las herramientas para utilizarlas. Empleando una analogía en el ámbito de la arquitectura, se podría decir que las pautas de accesibilidad son las normas en cuanto a las medidas de los edificios, sus accesos, la anchura de sus puertas o los servicios sanitarios requeridos, mientras que la ayuda técnica es la silla de ruedas que permite a la persona con discapacidad utilizar el edificio.

\section{INICIATIVA DE ACCESIBILIDAD WEB - WAI}

La Iniciativa de Accesibilidad Web (wAI-o6) es el grupo del w3 3 responsable de la accesibilidad. Está formado por profesionales internacionales de todos los ámbitos de actuación: autores, desarrolladores de software, desarrolladores de especificaciones, instituciones, empresas, etc. y su principal labor consiste en satisfacer las

necesidades de las personas con discapacidad.

En mayo de 1999 publicaron la primera versión de las Pautas de Accesibilidad para Contenido Web r.o (Web Content Accessibility Guidelines - WCAG I.O). Estas pautas, que presentan un modelo para la accesibilidad de la web, son las más extendidas y utilizadas, y a pesar de no ser un estándar, son la referencia indiscutible en Europa.

Además de las WCAG I.o, la WAI ha especificado técnicas, puntos de verificación detallados y casos de uso para la accesibilidad de las herramientas de autor, aplicaciones de usuario y documentos etiquetados XML, concretamente las pautas desarrolladas hasta el momento son: -Pautas de accesibilidad para contenido web I.o (Web Content Accessibility Guidelines r.o - wCAG I.o). Desarrolladas por el w3C en mayo de 1999, explican cómo hacer accesibles los sitios web para personas con diferentes tipos de discapacidad. Son catorce pautas de diseño, que incluyen puntos de verificación (checkpoints) que explican cómo aplicarlas y tienen un nivel de prioridad (de I a 3 ) asociado, en función de su importancia para la accesibilidad. El cumplimiento de los niveles determina tres niveles de accesibilidad finales posibles: A (el menos exigente), AA y AAA (el más restrictivo).

-Pautas de accesibilidad para contenido web 2.0 (Web Content Accessibility Guidelines 2.0 (working draft)). Estas nuevas pautas tratan de ser una actualización, de cara a las nuevas tecnologías desarrolladas desde la publicación de la versión r.o y a las que se desarrollen en un futuro, y solucionar los problemas surgidos con la versión anterior. En particular, se pretende no ligar las pautas a una tecnología específica, sino describir pautas lo suficientemente generales para aplicarlas en un contexto más amplio. También se redefinen 
los niveles de prioridad a dos: núcleo y extendido (core y extended). Las WCAG 2.0 se basan en cuatro principios generales que indican que las páginas web tienen que ser operables, comprensibles, robustas y se tienen que poder percibir por todos los usuarios.

-Pautas para la accesibilidad de las herramientas de autor 1.o (Authoring Tools Accessibility Guidelines r.o). Dirigido a desarrolladores de software, estas pautas describen cómo hacer que las herramientas de autor generen contenido web accesible, además de hacer que la propia herramienta sea accesible.

-Pautas para la accesibilidad de las aplicaciones de usuario r.o (User Agent Accessibility Guidelines 1.0). Dirigido a desarrolladores de software, explican cómo hacer accesibles los navegadores, los reproductores multimedia y la tecnología asistida que interactúa con estos programas.

- Pautas para la accesibilidad de documentos xмL (xмL Accessibility Guidelines). Dirigido a desarrolladores de aplicaciones basadas en XML, describen cómo conseguir que estas aplicaciones favorezcan la accesibilidad.

\section{I Seción 508}

La Sección 508 es parte de una ley de los Estados Unidos aprobada en 1998, que se basa en el Rehabilitation Act de 1973. Su principal objetivo es «proporcionar acceso electrónico y permitir la utilización de la información de las agencias federales ejecutivas a las personas con discapacidad» (Sección 2006). En ella se describen los requisitos mínimos de accesibilidad que deben cumplir las páginas web desarrolladas o utilizadas por las agencias federales de los Estados Unidos.

Con esta sección, el Comité para el Cumplimiento de las Barreras Arquitectónicas y de Transporte (Architectural and Transportation Barriers Compliance Board - Access Board) desa- rrolló estándares de accesibilidad para las diversas tecnologías que cubre la ley. El resultado de este proceso fueron los Estándares de Accesibilidad de las Tecnologías Electrónicas y de la Información (Electronic and Information Technology Accessibility Standards), que se publicaron en el Registro Federal en diciembre de 2000.

Los estándares del Comité de Accesibilidad son los únicos estándares para tecnología accesible que se han basado en una ley. Cubren un amplio abanico de estándares en tecnología electrónica y de la información, incluyendo, pero no limitado, a la tecnología Web.

Dentro de estos estándares se encuentra la sección titulada «Aplicaciones e Información en intranets o internets basadas en web», que incluye 16 estándares similares a los puntos de verificación de prioridad I dictados por la WAI. Se pueden ver las principales diferencias en la comparativa realizada por Jim Thatcher, Side-by-side comparison (wCAG vs. Sect. 508) (Thatcher 2005). Aunque en muchos aspectos la Sección 508 y las WCAG I.o son similares, en general las pautas de la WAI proporcionan un mayor nivel de accesibilidad.

\subsection{Ley 34/2002, de II de julio, de servicios de la sociedad de la información y de comercio electrónico (LSSICE)}

La LSSICE (BOE-O2) supone la primera regulación legal que con carácter general se dicta en España para el entorno de Internet.

Es de especial interés su disposición adicional quinta, en la cual se expresa la necesidad de «accesibilidad para las personas con discapacidad y de edad avanzada a la información proporcionada por medios electrónicos.»

En esta misma disposición se hace referencia a las obligaciones a cumplir por las administraciones públicas, concretamente dice que: «las Administraciones Públicas adoptarán las 
medidas necesarias para que la información disponible en sus respectivas páginas de Internet pueda ser accesible a personas con discapacidad y de edad avanzada de acuerdo con los criterios de accesibilidad al contenido generalmente reconocidos antes del 3I de diciembre de 2005 . Asimismo, podrán exigir que las páginas de Internet cuyo diseño o mantenimiento financien apliquen los criterios de accesibilidad antes mencionados».

\subsection{Ley 5I/2003, de 2 diciembre, de Igualdad de Oportunidades, No Discriminación y Acccesibilidad Universal (LIONDAU)}

La Ley de Igualdad de Oportunidades, No Discriminación y Accesibilidad Universal (LIONDAU) (воE: O3), publicada en diciembre de 2003 , tiene entre sus principales objetivos, descritos en el capítulo I de la ley, asegurar la vida independiente, la normalización, la accesibilidad universal, el diseño para todos, el diálogo civil y la transversalidad. Entre sus ámbitos de aplicación, figuran expresamente las telecomunicaciones y sociedad de la información, lo cual obliga a las administraciones públicas y demás empresas que ofrecen sus servicios a través de Internet, a no discriminar a las personas por el hecho de sufrir algún tipo de discapacidad.

\subsection{Anteproyecto de ley de infracciones y sanciones en materia de igualdad de las personas con discapacidad}

E1 Consejo de Ministros (Moncloa 2006) recibió un Informe del Ministro de Trabajo y Asuntos Sociales (MTAS 2005) sobre el Anteproyecto de ley de infracciones y sanciones en materia de igualdad de oportunidades, no discriminación y accesibilidad universal de las personas con discapacidad. Este Anteproyecto de Ley tiene como objetivo establecer un régimen

sancionador eficaz ante las infracciones que vulneren la Ley de igualdad de oportunidades, no discriminación y accesibilidad universal de las personas con discapacidad.

$Y$ en él se considerará infracción administrativa: «Las vulneraciones del derecho a la igualdad de oportunidades, no discriminación y accesibilidad universal de las personas con discapacidad cuando se produzcan discriminaciones directas o indirectas, acosos, incumplimiento de las exigencias de accesibilidad y de realizar ajustes razonables, así como el incumplimiento de las medidas de acción positiva legalmente establecidas, especialmente cuando se deriven beneficios económicos para el infractor».

\subsection{Anteproyecto de ley de lengua de signos y de apoyo a la comunicación oral}

En el mismo Consejo de Ministros (Moncloa 2006) se aprobó un anteproyecto, pionero en Europa, de lengua de signos y de medios de apoyo a la comunicación. La norma reconocerá la lengua de signos española como lengua de las personas sordas en España que libremente decidan utilizarla, así como su aprendizaje, conocimiento y uso. Además, establecerá y garantizará los medios de apoyo a la comunicación de las personas sordas, con discapacidad auditiva y sordociegas.

Concretamente, en el anteproyecto se establece que:

«I. España reconocerá legalmente la lengua de signos e impulsará los sistemas de apoyo a la comunicación.

2. Las administraciones educativas facilitarán el aprendizaje de la lengua de signos española y de los sistemas de apoyo a la comunicación.

3. Las personas sordas y con discapacidad auditiva podrán solicitar intérpretes y medios de apoyo a la comunicación en los servicios básicos. » 


\subsection{Legislación europea}

Una de las primeras referencias a la accesibilidad y a la no discriminación en la legislación europea, aparece en el artículo I3 del Tratado de Ámsterdam de 1997, en el que se menciona la prohibición de la discriminación por discapacidad (Euro 1999).

Las directivas de telecomunicaciones, de 1997, tienen entre uno de sus objetivos garantizar el servicio universal y la protección de datos. Pero la referencia más clara de la responsabilidad de los estados miembros y administraciones aparecen en la Resolución del Consejo de la Unión Europea relativa a la integración social mediante tecnologías electrónicas (Consejo de la Unión Europea: 2006), de octubre de 200I, en el que se indica que «los estados miembros deberán asegurar el acceso a tecnologías electrónicas fáciles y asequibles». Se puede apreciar el hecho de que no se pide la promoción o el favorecimiento de estas tecnologías, sino que se exige la seguridad de proporcionar un acceso a las tecnologías.

En la Resolución del Consejo de la Unión Europea sobre el plan eEuropa 2002: accesibilidad a los sitios web y sus contenidos, de marzo de 2002 , se recoge la necesidad de que «las administraciones públicas nacionales deberán mejorar la accesibilidad de sus páginas web» y se reconocen por primera vez las pautas WAI para la accesibilidad de sitios web, que posteriormente se extenderán como estándar de facto en toda Europa.

Por último, el plan más reciente a nivel europeo es el plan eEuropa 2005, una sociedad de la información para todos, de 2004, que tiene como objetivo «asegurar los servicios públicos accesibles que exploten posibilidades de banda ancha y acceso multiplataforma».

\subsection{Otras normas}

La Organización Internacional para la Estandarización (Iso) ofrece la nueva especificación técnica Iso/Ts I607i Ergonomía de la comunicación hombre/sistema. En ella proporciona directrices para que el diseño de software sea accesible y pueda conectarse y relacionarse con herramientas de tecnología asistida.

Por otro lado, las normas UNE elaboradas por los Comités Técnicos de Normalización de AENOR proporcionan, para su uso común y repetido, reglas, directrices o características para ciertas actividades o resultados.

- La norma UNE I3980r:2003 contiene 59 requisitos para aplicaciones informáticas para personas con discapacidad. Requisitos de accesibilidad al ordenador. Hardware.

- La norma UNE 139802:2003 contiene 90 requisitos para aplicaciones informáticas para personas con discapacidad. Requisitos de accesibilidad al ordenador. Software.

Actualmente se está trabajando en la «Norma de Internet: PNE 139803 Requisitos de accesibilidad para contenidos en la web». Que será una versión actualizada y ampliada del capítulo «Requisitos de accesibilidad del acceso hipermedia a las autopistas de la información».

Los futuros trabajos del subcomité AEN/CTN I39/SC8 se centran en agrupar los requisitos por prioridad, clasificarlos en función del tipo de producto y su relación con cada tipo de discapacidad.

\section{USABILIDAD}

La accesibilidad implica que las personas con discapacidad puedan acceder a contenidos o servicios, a través de tecnologías asistidas, si fuese necesario. La usabilidad, sin embargo, entendida en el ámbito de las Tic, significa que las personas que las utilizan lo hacen de forma satisfactoria, utilizando para ello tecnología asistida, si fuese necesario. 
Según Jakob Nielsen (1993) «la usabilidad es la cualidad de un sistema por la que resulta fácil de aprender, fácil de utilizar, fácil de recordar, tolerante a errores y subjetivamente placentero.»

La Iso 924I-II proporciona una definición en la que se mencionan las tres palabras claves de la usabilidad, a saber: eficiencia, eficacia y satisfacción. «La medida en la que un producto se puede usar por determinados usuarios para conseguir objetivos específicos con efectividad, eficiencia y satisfacción en un contexto de uso» (ISO I998).

No siempre existe una preocupación por lograr diseños e interfaces usables. Existen comentarios y opiniones que consideran la usabilidad como algo subjetivo difícil de medir, que no es necesario tener en cuenta hasta fases avanzadas del proyecto o que son suficientes los conocimientos de los ingenieros del software para diseñar una buena interfaz. Estas creencias llevan a menudo a cometer errores en la interfaz que son difíciles de corregir si el proyecto está muy avanzado.

El diseño accesible no beneficia únicamente a las personas con discapacidad, sino que incrementa el nivel global de usabilidad. A menudo, una vez logrado el certificado de accesibilidad deseado o pasado un validador automático se da por finalizada la verificación de la accesibilidad, no se analiza si la interfaz y diseños desarrollados son amigables o no, si son fáciles de utilizar o si existen elementos que no se pueden verificar automáticamente. Hay que tener en cuenta que los puntos de verificación se pueden cumplir sin que ello implique un diseño accesible y usable. Por ejemplo, el texto alternativo a un mapa geográfico de La Rioja podría ser «mapa», lo cual si bien cumple las pautas de accesibilidad dice bien poco de la información visual que se transmite con la imagen.
Leporini (Leporini: 04) propone 16 criterios para incrementar la usabilidad de las páginas web a las que se accede mediante lectores de pantalla. Estos criterios se dividen en pautas de presentación, que indican cómo la interfaz proporciona información al usuario, y pautas de diálogo, que describen la forma en la que se secuencializan las acciones del usuario y del sistema.

Así como se han desarrollado múltiples pautas de accesibilidad, centradas en proporcionar acceso a las personas con discapacidad, también existen pautas, técnicas y recomendaciones para incrementar la usabilidad de los sitios web. Los principios del Diseño para Todos o, como es llamado en Estados Unidos, Diseño Universal, fueron compilados por: Bettye Rose Connell, Mike Jones, Ron Mace, Jim Mueller, Abir Mullick, Elaine Ostroff, Jon Sanford, Ed Steinfeld, Molly Story y Gregg Vanderheiden (Connell et al 1997). Aunque algunos de los principios están estrechamente ligados a las pautas WCAG, que ya han sido descritas, otros van más allá y describen los mecanismos y estructuras de navegación y las pautas diseño, estructura y organización del contenido más adecuadas.

Este grupo de arquitectos, diseñadores e ingenieros del Centro de Diseño Universal de la Universidad de Carolina del Norte, describieron en 1997 los siete principios del diseño universal. Estos principios, que describen las características de un diseño universal usable, son:

-Uso equitativo. Proporcionar información equivalente a todos los usuarios, idéntica cuando sea posible, evitando la discriminación y asegurando la seguridad y privacidad.

-Flexibilidad en el uso. El diseño se debe adaptar a las preferencias y habilidades de un amplio grupo de usuarios.

-Uso sencillo e intuitivo. El diseño debe 
ser fácil de entender, independientemente de la experiencia del usuario, sus conocimientos, habilidades o nivel de concentración.

- Información percibida. El diseño comunica la información al usuario de manera eficiente, independientemente de las condiciones ambientales o las habilidades sensoras del usuario.

- Tolerancia a errores. Minimizar los peligros y consecuencias negativas de los errores producidos de forma accidental o no intencionada.

-Bajo esfuerzo físico. El diseño se puede utilizar de forma eficiente y confortable con un mínimo esfuerzo.

- Tamaño y espacio de aproximación y utilización. Se debe proporcionar un espacio y tamaño adecuados para la aproximación, alcance, manipulación y utilización, independientemente del tamaño, postura o movilidad del individuo.

\section{Conclusiones}

Para avanzar en el área de la accesibilidad y usabilidad es fundamental conocer los tipos de discapacidad que afectan al uso de las TIC, la problemática concreta de cada una de ellas y las soluciones existentes.

Las discapacidades sensoriales, y entre ellas la ceguera, son las más conocidas, pero no las únicas. La discapacidad motora, cognitiva o los problemas del habla son otro tipo de discapacidad que dificultan o impiden el acceso a las TIC.

Existen además falsas creencias en cuanto a la problemática de las personas con discapacidad, por ejemplo, el principal problema de una persona con discapacidad auditiva frente a las TIC puede parecer el hecho de oír con dificultad, pero su gran problema es la comunicación y comprensión. Es importante además, conocer las posibilidades de estos colectivos, qué es lo que sí pueden hacer, para fomentarlo y favorecerlo.

Las ayudas técnicas son productos y tecnolo167 gías, dirigidas a usuarios con discapacidad, que permiten o facilitan el acceso a los dispositivos hardware, $y$ a los servicios y contenido ofrecidos a través de diferentes programas informáticos.

Las ayudas existentes para los diseñadores y desarrolladores tecnológicos son las pautas, técnicas y recomendaciones de accesibilidad. El referente europeo, y estándar de facto, son las normas WCAG I.o de la WAI, organismo dependiente del w3c. En cualquier caso hay otras normas menos conocidas, que afectan a cualquier tipo de software e interfaz de usuario.

La legislación es uno de los factores que más impulsan el desarrollo de tecnología accesible. La Sección 508 estadounidense ha supuesto que en EE.UU. haya una mayor preocupación en las empresas por la accesibilidad, esto mismo se está consiguiendo en Europa y en España con planes como eEuropa y leyes como la «Ley de servicios de la sociedad de la información y de comercio electrónico — LSSICE», «Ley de Igualdad de Oportunidades, No Discriminación y Acccesibilidad Universal - LIONDAU» o el «Anteproyecto de ley de infracciones y sanciones en materia de igualdad de las personas con discapacidad».

Es importante darse cuenta de que no hay una única interfaz que satisfaga a todos los usuarios, se deberían proporcionar interfaces alternativas, o al menos dar la opción de personalizarlas, para adaptarse a las limitaciones, habilidades, requisitos y preferencias de los usuarios.

Stephanidis (Ioor) propone tres requisitos para cumplir este objetivo, que son: enumerar las posibles alternativas en cuanto a diseño de 
la interfaz, intentando acomodarse al mayor número posible de usuarios y proporcionando diferentes opciones; encapsular las alternativas 168 en un nivel de abstracción que describa de forma independiente el interfaz de la realización física del mismo; y racionalizar el espacio resultante de diseño, relacionando el diseño abstracto obtenido anteriormente con la realización física del mismo, evaluar las diferentes alternativas y determinar cuándo una es mejor que la otra.

Por último, es importante ver la accesibilidad como algo más global que el hecho de poder acceder a las TIC, bien desde el punto de vista hardware como software. Los requisitos que tienen todos los usuarios en cuanto a eficiencia, eficacia y satisfacción en el uso de las Tic, también se debe contemplar a la hora de ofrecer tecnología a las personas con discapacidad. Esto se engloba en un término más amplio, que es usabilidad.

\section{RECIBIDO EN DICIEMBRE 2006 ACEPTAdo EN ENERO 2007}

\section{BIBLIOGRAFÍA}

Abascal, Julio (1998): «Informática y discapacidades». Novática $136:$ 2-5.

Adaptative Techology Resource Center - atrc. University of Toronto. 2006. En http://atrc. utoronto.ca

Adaptative Techology Resource Center - atrc. University of Toronto. APrompt, Web Accessibility Verifier. 2006. En http://aprompt.snow.utoronto. ca

Berners-Lee, T., Fischetti, M. (1999): Weaving the Web: The Original Design and Ultimate Destiny of the World Wide Web by Its Inventor. Harper: San Francisco.

Boletín Oficial del Estado - воE. Ley 34/2002, de II de julio, de servicios de la sociedad de la información y de comercio electrónico. Boletín Oficial del Estado. BOE $\mathrm{n}^{\circ} \mathrm{I} 66$ del viernes I2 de julio de 2002.

Boletín Oficial del Estado - воE. Ley 5I/2003, de 2 de diciembre, de igualdad de oportunidades, no discriminación y accesibilidad universal de las personas con discapacidad. Boletín Oficial del Estado. вов $\mathrm{n}^{\circ} 289$ del miércoles 3 de diciembre de 2003 .

Connell, BR. et al (Ed.) (1997): The Principles of Universal Design. Version 2.0 - 4/1/97. Raleigh, NC: NC State University.

Fundación cTic, Centro Tecnológico de la Información y Comunicación. Test de Accesibilidad Web, TAW. 2006. En http://www.tawdis.net

Consejo de la Unión Europea. 2006. En http://www. consilium.europa.eu

Euro-99, El portal de la Unión Europea. J. (1999): Tratado de Amsterdam por el que se modifican el Tratado de la Unión Europea, los Tratados constitutivos de las Comunidades Europeas y determinados actos conexos. I999. En http://europa.eu/eur-lex/ es/treaties/selected/livre546.html

Freedom Scientific. JAWS (C) for Windows. 2003. En http://www.freedomscientific.com

(GWM-06) GW Micro, Inc. Window Eyes. 2004. En http://www.gwmicro.com

(HiSo-o6) HiSoftware. AccVerify. 20o6. En http:// www.hisoftware.com

(гвм-о6) гвм Corporation. 2006. En http://www. ibm.com

(IвRC-06) International Braille Research Center IBRC. 2006. En http://www.braille.org

(ISC-o6) Internet Systems Consortium. Lynx text Web browser. En http://lynx.isc.org

(Iso-98) International Standarization Organization, ISO. ISO 924I-II:I998 Ergonomic requirements for office work with visual display terminal (VDTs)Part II: guidance on usability. I998.

B. Leporini y F. Paternò (2004): «Increasing Usability when Interacting through Screen Readers» Springer International Journal Universal Access in the Information Society (UAIS) Special Issue on Guidelines, Standards, Methods and Processes for Software Accessibility, (3) r: 57-70.

Moncloa, La. Consejo de Ministros. En http://www. la-moncloa.es

MTAS- Ministerio de Trabajo y Asuntos Sociales. III Plan Nacional de Acción para la Inclusión Social del Reino de Expaña 2005-2006. 8-septiembre2005 .

Nielsen, J. (I993): Usability Engineering. Academic Press Professional, Boston, MA. 
oms - Organización Mundial de la Salud - oms. International Classification of Functioning, Disability and Health (ICF). $200 \mathrm{I}$.

Section508.com, Inc. 2006. At http://www.section508.gov

SIDAR- Seminario Iberoamericano sobre Discapacidad y Accesibilidad en la Red - SIDAR. Hera. 2006. At http://www.sidar.org/hera

Stephanidis, C. (200I): User interfaces for all. Concepts, Methods and Tools. Lawrence Erlabum Associates, Publishers.

Tegic Communications, Inc. 2006. En http://www. t9.com
Thatcher, J. (2005): Side-by-side comparison (WCAG vs. Sect. 508).En http://www.jimthatcher.com/sidebyside.htm

Vanderheiden, G. C. (1998): «Presente y futuro de la accesibilidad a Internet» Novatica I36: 6-II. w3c. En http://www.w3.org/QA/Tools/ \#validators

(WAI-o6) Web Accessibiliy Initiative - wAI. 2006. En t http://www.w3c.org/wai

(Watc-02) Watchfire Corporation. Bobby Online Free Portal. 2002. En http://bobby.watchfire.com 